\title{
Interactive testbed for research in autism-the SARA project
}

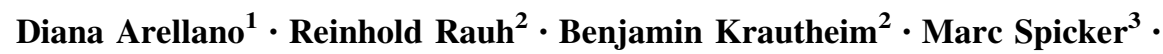 \\ Ulrich Max Schaller ${ }^{2} \cdot$ Volker Helzle ${ }^{1} \cdot$ Oliver Deussen $^{3}$
}

\begin{abstract}
The project Stylized Animations for Research on Autism (SARA) aims to develop a better understanding of the cognitive processes behind emotional categorization in children and adolescents with high-functioning autism spectrum disorder (ASD), in comparison with neurotypically developed peers. To this end, we combine novel realtime non-photorealistic rendering (NPR) algorithms, emotional facial animations, and eye-tracking technologies in a framework that serves as an interactive testbed for empirical research. In this paper, we focus on three experiments that: (1) validate real-time facial animations of virtual characters, (2) evaluate the NPR algorithms to create abstracted facial expressions, and (3) elucidate the relation between eye gaze behavior, ASD and alexithymia (i.e., difficulties in expressing ones emotions). The results show that our animations can be used in the proposed experiments; however, more evaluation is needed regarding the NPR abstractions, especially with individuals with ASD. Finally, even though no correlation was found between gaze behavior, ASD and alexithymia, the study opened several questions that will be addressed in future work.
\end{abstract}

Diana Arellano and Reinhold Rauh have contributed equally to this work.

Diana Arellano

diana.arellano@filmakademie.de

1 Animationsinstitut at Filmakademie Baden-Wuerttemberg, Ludwigsburg, Germany

2 Department of Child and Adolescent Psychiatry, Psychotherapy, and Psychosomatics, Medical Center University of Freiburg, Faculty of Medicine, University of Freiburg, Freiburg, Germany

3 Chair of Computer Graphics and Media Informatics, University of Konstanz, Konstanz, Germany
Keywords Autism spectrum disorder · Facial animation . Eye tracking $\cdot$ Stylization

\section{Introduction}

ASD is a developmental disability that tosses significant communication, behavioral and social challenges. According to the Center for Disease Control and Prevention (CDC), research on ASD has increased a great deal in recent years [1], as well as the number of children and adults with this disorder.

In 2014, the CDC estimated that about one percent of the world population had ASD. In the USA, the prevalence of ASD was in the same year one in 68 births [2]. More recently, the prevalence rate in Europe indicates that 1 in 100 people has this disorder [3, 4].

Motivated by these numbers and the amount of research done in this area [5, 6], we proposed SARA, a project that combines clinical psychology, NPR, 3D facial animation and eye-tracking technology. The main objective is to investigate the causes behind communication and emotion perception deficits in children and adolescents with highfunctioning ASD.

In this paper, we present a compilation of three experiments carried out during the course of SARA. In all them, a set of virtual characters displaying emotional facial animations are used in an interactive computer-based psychological test. All animations are generated in real time. The first experiment, R-DECT, assesses one important facet of "rapid social cognition" of children and adolescents with ASD.

The second experiment, NPR-DECT, served as a pilot study in order to appraise how NTD individuals categorize abstracted faces with reduced levels of detail. The third 
experiment is part of the NPR-DECT, but focused on determining the differences in eye gaze behavior between NTD and ASD participants. More specifically, we want to better ascertain the relation between ASD, gaze behavior, alexithymia and deficits in facial emotion perception. In this study, we only evaluated gaze behavior during the interaction with the realistic-looking versions of the characters. The remaining NPR-DECT gaze data (i.e., when interacting with the abstracted characters) will be further analyzed, but does not belong to the scope of this paper.

One of the main interests of this project is to better understand the cognitive processes behind the categorization of emotions and eye gaze behavior in ASD. This is crucial to designing and implementing methodologies and computer-based interventions (CBI) that can assist them in the improvement of social skills. Additionally, the use of a real-time interactive environment broadens the possibilities for experimentation and research that otherwise would not be possible (e.g., with pre-rendered animations).

In the following, we will refer to previous works on character facial animation and gaze behavior on autism research. Then, we will explain the SARA project and the tools developed and used in it. The subsequent sections unfold each of the three experiments, providing details on the participants, stimuli, procedure and measures. Finally, a discussion of the results of the experiments is given, followed by our conclusions and future work.

\section{Related work}

Many interactive applications created to develop or enhance the social skills of individuals with autism make use of virtual characters. For instance, Whyte et al. [7] used game components (e.g., storyline, long-term goals, rewards) to create engaging learning experiences. Milne et al. [8] employed autonomous agents as tutors for teaching children with ASD conversation skills, and how to deal with bullying. Grawemeyer et al. [9] developed a sort of non-photorealistic embodied pedagogical agent together with and for young people with ASD.

ECHOES VE [10] presented a virtual environment where children with ASD need to assist a cartoony virtual character in selecting objects by following the character's gaze and/or pointing at the object. JeStiMulE [11] attempted to teach participants to recognize emotions on the faces and gestures of virtual characters, while considering the context. LIFEisGAME [12] deployed a low cost real-time animation system embedded in a game engine to create a game that helps individuals with ASD to recognize emotions in an interactive way. FaceSay ${ }^{\mathrm{TM}}$ [13] aided children with ASD to recognize faces, facial expressions and emotions by offering students simulated practice with eye gaze, joint attention and facial recognition skills. Let's face it! [14] was a set of seven interactive computer games targeting the specific face impairments associated with autism.

A common element in all of these applications is the use of virtual characters to enhance or develop skills in subjects with ASD. These characters presented a defined visual style, which was either cartoony or realistic. In this sense, one of the assets of our project is the wider spectrum of real-time stylizations it provides. They resemble more artistic styles, which maintain as far as possible the humanlike features of the virtual characters' faces. By offering varied painterly representations with different levels of abstraction, the experimenters can change the visual representation of the characters in real time, achieving more personalized applications.

Regarding the study of gaze behavior, normally these applications do not employ eye-tracking (even though, it could be adapted) to assess irregularities in eye contact and gaze behavior in participants with ASD. The DSM-5 [15] and ICD-10 [16], the criteria used by clinicians and researchers to diagnose and classify mental disorders, lists abnormalities in eye contact as one possible sign of deficient nonverbal communication patterns in people with autism. However, the findings on ASD and gaze avoidance have been inconsistent so far [17].

Kirchner et al. [18] found, for instance, that people with ASD fixated less on the face than NTD participants, while taking the Multifaceted Empathy Test (MET).

Klin et al. [19] carried out a study where high-functioning ASDs and NTDs had to watch naturalistic social scenes while eye gaze was tracked. They observed that a reduction in eye region fixation time serves as the best predictor of ASD. Apart from that, individuals with highfunctioning ASD and improved social adjustment skills showed increased fixation duration on mouths.

Senju and Johnson [20] explored reduced eye contact in ASD, distinguishing four models of atypical eye contact: (1) hyperarousal model: ASDs perceive the eyes of others as aversive stimuli; (2) hypoarousal model: gaze behavior stems from a hypoactivation of the amygdala in early childhood; (3) communicative intention detector model: atypical eye contact in ASD reposes on the difficulty in reading others mental states due to impairments inferring mentalistic significance of the eyes; and (4) fast track modulator model: ASDs are impaired with regard to the subcortical face detection pathway. Therefore, they perform more poorly when pictures or videos of faces are presented for a short time span, or when they contain low spatial frequency information.

In contrast, Sawyer et al. [21] demonstrated that individuals with Asperger's syndrome showed no gaze avoidance in comparison with participants with ASD. 
A possible solution to this debate was offered by Bird et al. [22]. They hypothesized and proved that the degree of alexithymia predicts deviant gaze behavior in ASD patients. In a subsequent work, Bird and Cook [23] went a step further to argue that emotional deficits in ASD might actually be due to alexithymia instead of ASD symptomatology per se.

Our study nurtures from these previous hypotheses, and attempts to shed new light on the connection between autism, gaze behavior and recognition of facial emotions.

\section{SARA: Stylized Animations for Autism Research}

In order to evaluate the categorization of dynamic emotional facial expressions by children and adolescents with high-functioning ASD, SARA combines clinical psychology, computer graphics and 3D animation.

One of the main innovations includes the use of realtime NPR algorithms to abstract the faces of the virtual characters used in the various test. This allows us to explore how a reduction in the levels of detail of facial expressions affect their categorization by individuals with ASD.

On its part, eye-tracking technologies allow for the study of gaze behavior in ASD, giving the possibility to extend and corroborate the results of previous studies with an adolescent sample.

\subsection{DECT: Dynamic Emotion Categorization Test}

The core of SARA is the DECT [24], an interactive computer-based tool created to determine the feasibility of using real-time animations and to assess dynamic emotion categorization in facial expressions.

The original test contained material of two human actors and two virtual characters displaying dynamic facial expressions of the basic emotions: anger, disgust, fear, happiness, sadness and surprise. These were presented on three intensity levels: weak, medium and strong. The results showed that facial animations in virtual characters presented good concurrent validity with video clips of human actors in general. Furthermore, using parameterizable facial animations of virtual characters provided for an easier way to adjust emotion intensity levels in comparison with human actors. This motivated us to further explore the use of virtual characters in autism research.

Currently, the interaction with the software has been designed in a way where the experimenter has more control over it. Thanks to the real-time and parameterizable characteristics of the animations and NPR styles, the experimenter can generate different trials by direct manipulation of

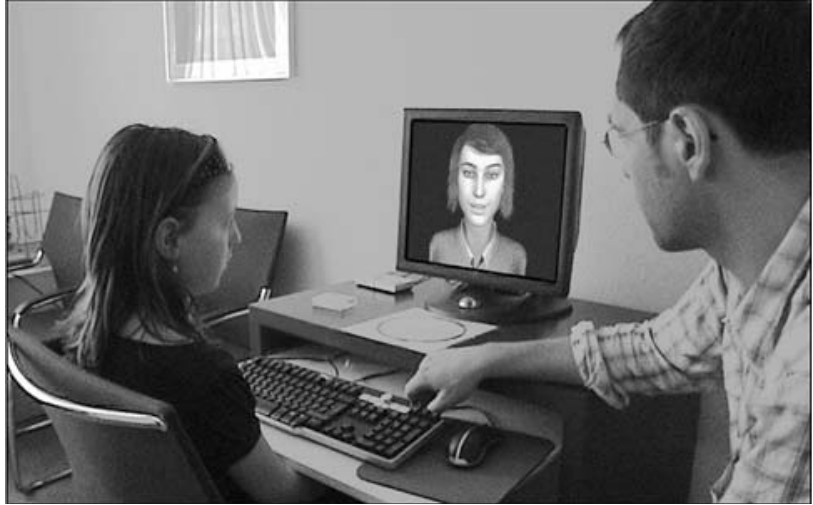

Fig. 1 Participant during a DECT session

these elements. During the practice trials, the experimenter explains to the participant what needs to be done (Fig. 1).

The test deliberately does not contain any GUI-centric terms, so participants with ASD do not focus on other elements than the facial expressions of the characters and the answer options with emotion names.

\subsection{Frapper: Filmakademie Application Framework}

The development of the current DECT versions (R-DECT and NPR-DECT), as well as the implementation of the NPR algorithms and real-time animations, was done using our in-house software development platform Frapper, ${ }^{1}$ particularly the Agent Framework [25].

Frapper is a C++-, Ogre3D- and Qt-based development environment consisting of a node-based scene model, a model-view-controller architecture and a panel-oriented user interface similar to commercial 3D packages. The Agent Framework is the set of functionalities (nodes and plugins) that allow users the rapid prototyping of applications that make use of virtual characters.

New functionality can be simply added by creating node or panel plugins, which incorporate the desired features and allows linking to third-party libraries. In this way, complex technologies can be brought into Frapper, such as eye tracking, computer vision, synthetic speech, voice recognition or artificial intelligence, as well as the integration of alternative input devices.

Frapper is available as open source and is released under the GNU Lesser General Public License 2.1.

\subsection{Animated virtual characters}

All characters were created to resemble a realistic-looking person. For the studies presented in this paper, we used two

\footnotetext{
${ }^{1} \mathrm{http} / / /$ sourceforge.net/projects/frapper/.
} 
characters: an elderly man, named Hank, and a young woman, named Nikita.

These were rigged using the Adaptable Facial Setup (AFS) [26], a tool set that relies on a complete motion capture-based library of deformations, based on the Facial Animation Coding System (FACS) [27], generating high-quality, natural and nonlinear deformations. Once the characters were finished, they were exported into the Animation Framework. The facial movements are described in terms of action units (AUs), providing a parameterizable way of creating animated facial expressions, which could then be translated to any other character with a similar FACS-based rig.

Both Frapper and the Agent Framework are provided with the two humanlike characters distributed under the Creative Commons Attribution Non-Commercial Share Alike 3.0 Unported License.

\subsection{Interactivity}

A relevant aspect of the project is the level of interactivity it provides thanks to the real-time characteristics of our framework. Having a tool that generates animations and abstract visual representations in real time allows the psychologists and experimenters to fine-tune and parameterize the tests themselves. This results in more flexible and elaborated interactive experiments, which can be adapted to the experimenter's requirements, or participant's needs. It also makes the experimenters independent from the animator, an important aspect to consider when using a computer-based research tool.

\section{DECT session}

A session with both versions of DECT (R-DECT and NPRDECT) consists of several trials, which in turn are composed by a series of elements displayed in Frapper (Fig. 2).

Frapper works in combination with the open-source software PsychoPy [28]. PsychoPy is a free, open-source

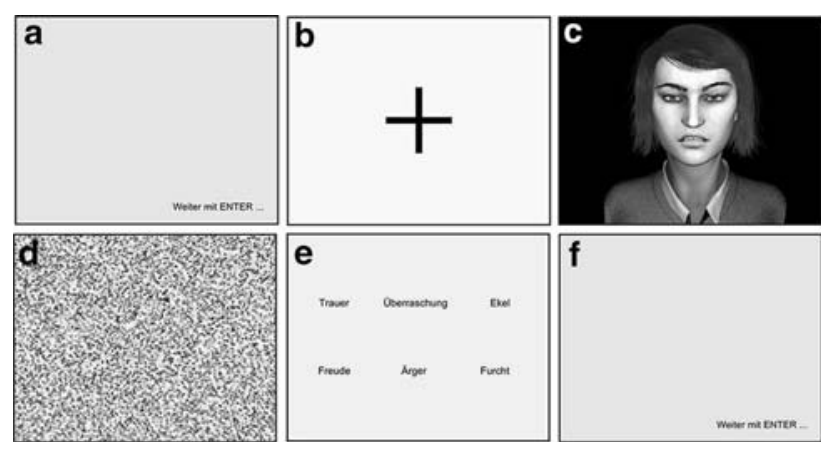

Fig. 2 DECT screens: a initial screen, $\mathbf{b}$ fixation cross, $\mathbf{c}$ character with an angry expression, d white noise, e forced choices with emotion names, $\mathbf{f}$ initial screen for next trial application written in Python. It allows to design and implement the logic of the experiments, along with the collection of data.

A trial begins with a pink-colored screen (Fig. 2a), which serves as separator between trials. By pressing the Enter key, the trial and the interaction is initiated.

The next element is a fixation cross (Fig. 2b), which appears for $0.5 \mathrm{~s}$ and indicates where the participant should fixate his gaze.

Afterward, either Hank or Nikita appears, displaying a real-time generated animation of a facial expression. In the case of the R-DECT, different intensity and speed levels were used (Fig. 2c). For the NPR-DECT, the intensity of the expression is always medium. Moreover, it extends the visual representation to one of the following NPR styles: original (realistic looking with no stylization), pencil drawing, coherent line drawing, image abstraction or watercolors. As for the level of abstraction, it can be low, medium or high.

Then, a screen with white noise (Fig. 2d) is displayed for $0.5 \mathrm{~s}$, masking participants' iconic memory with taskirrelevant information.

Finally, another screen with the answer options represented by emotional labels is displayed (Fig. 2e). Here the user needs to select the one corresponding to the expression that he just saw.

In order to select an emotion, each of the basic emotions was mapped to a number between 1-3 and 7-9, which was then selected using the numeric pad of the keyboard. The reason for not using the row 4-6 was to allow space between the fingers and avoid experimental errors by inadvertently pressing the wrong key. The pairing emotion number is constant within the experimental session, though counterbalanced across participants according to a sequentially counterbalanced latin square.

In the following, the R-DECT, NPR-DECT and the study on gaze behavior will be presented.

\section{Experiment 1: R-DECT}

R-DECT [29] is a DECT version that was part of a battery of tests for rapid social cognition and intuitive moral reasoning assessment in children and adolescents with ASD. It also served to validate the improved facial animation from the original DECT, as well as to validate the test itself as a tool for the interactive categorization of emotional expressions.

\subsection{Participants}

Our sample consisted of 39 adolescents between 14.0 and 17.9 years old, and IQ $\geq 70$. Two groups were considered: 
- The group of neurotypically developed adolescents (NTD group: $n=22$ ) consisted of 18 males and 4 females;

- The group of individuals with high-functioning ASD (ASD group: $n=17$ ) consisted of 12 males and 5 females.

\subsection{Stimuli}

For each session, 36 animations were evaluated: 2 (characters $) \times 6$ (basic emotions) $\times 3$ (intensity levels). The intensity levels included weak, medium and strong. The speed variable was assigned according to a certain scheme to each of the 36 animations, ranging from 1 (normal speed) up to 2.25 times of normal speed. In total, six levels were used: 1.00, 1.25, 1.50, 1.75, 2.00, 2.25.

\subsection{Procedure}

The rapid social cognition experiment comprised two sessions, each lasting between 1.5 and $2 \mathrm{~h}$. However, the R-DECT needed approximately $15 \mathrm{~min}$ to be carried out, where each trial had a duration of approximately $2 \mathrm{~s}$. The R-DECT was administered as the first of five tests in the corresponding session. The way each DECT session was carried out is explained in Sect. 4.

\subsection{Results}

In total, $62.2 \%$ of the animations were categorized correctly. The accuracy rate for the NTD group was $65.7 \%$, whereas for the ASD group $57.7 \%$.

A $2 \times 6$ MANOVA with repeated measurements showed no significant interaction between group and basic emotion $(F<1)$. However, the two main effects were significant (basic emotion: $F(5,33)=77.63, p<0.0001$; group: $F(1,37)=5.36, p=0.026)$. This indicated that the ASD group performed significantly worse than the NTD group (Fig. 3).

The order of accuracy for basic emotions was the same for both groups, where "happiness" was the one recognized with the highest accuracy and "fear" with the least. Similar results concerning order of accuracy rates of basic emotions were obtained in [30, 31].

None of the post-hoc comparisons reached statistical significance.

As for the intensity of facial emotions, we only considered the NTD group where typical facial emotion recognition is expected. The results showed that, in general, varying intensities from weak over medium to strong affected accuracy rates correspondingly: weak: $59.5 \%$, medium: $65.5 \%$ and strong: $72.0 \%$ [29].

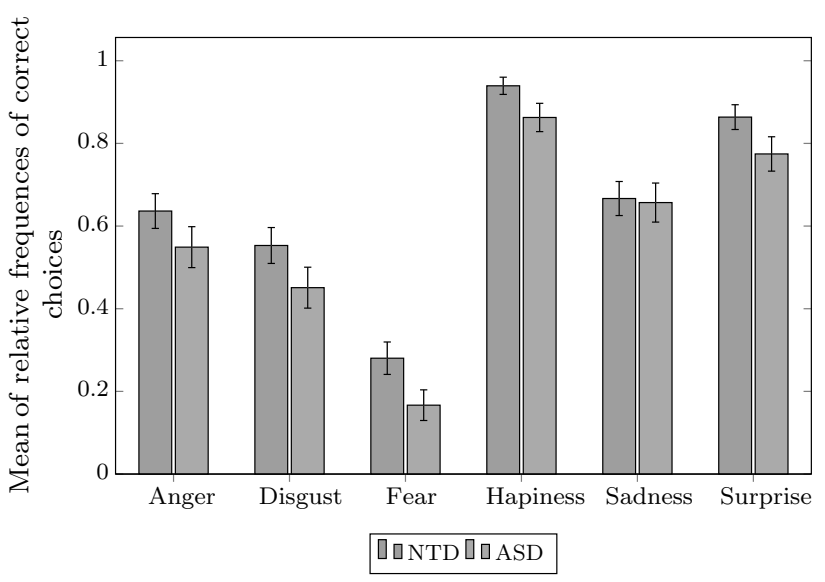

Fig. 3 Mean of relative frequencies of correct categorizations of six basic emotions in ASD and NTD groups. Error bars represent 95\% CI

\section{Experiment 2: NPR-DECT}

The second version, NPR-DECT, comprised one of the novelties of this project: the use of NPR algorithms to abstract and manipulate visual information in the faces of our virtual characters.

We used NPR to stylize the faces of the characters as it provides variation in the level of abstraction and visual information, adapting images to "focus the viewer's attention" [32]. Thus, the information load in the characters' facial expressions can be reduced, conveying the emotional information more efficiently [33, 34].

NPR-DECT was the way to include more artistic approaches to investigate how these abstractions affect the recognition of the facial expressions of emotions in comparison with their more realistic representations. In addition, this test served to study the link between alexithymia, ASD and deviant gaze behavior.

\subsection{Participants}

Our sample consisted of 31 NTD adults ( 9 males and 22 females) with ages between 20 and 35 years.

No subjects with ASD were considered, as the aim was to validate the developed NPR styles. The final experiment is described in [35].

\subsection{Stimuli}

The NPR-DECT comprised 2 (characters) $\times 6$ (basic emotions $) \times 13(1+12$ NPR style $\times$ abstraction combinations). This resulted in 156 trials, which were presented in a pseudorandomized order.

The NPR styles used (Fig. 4), apart from the original realistic looking, were: 

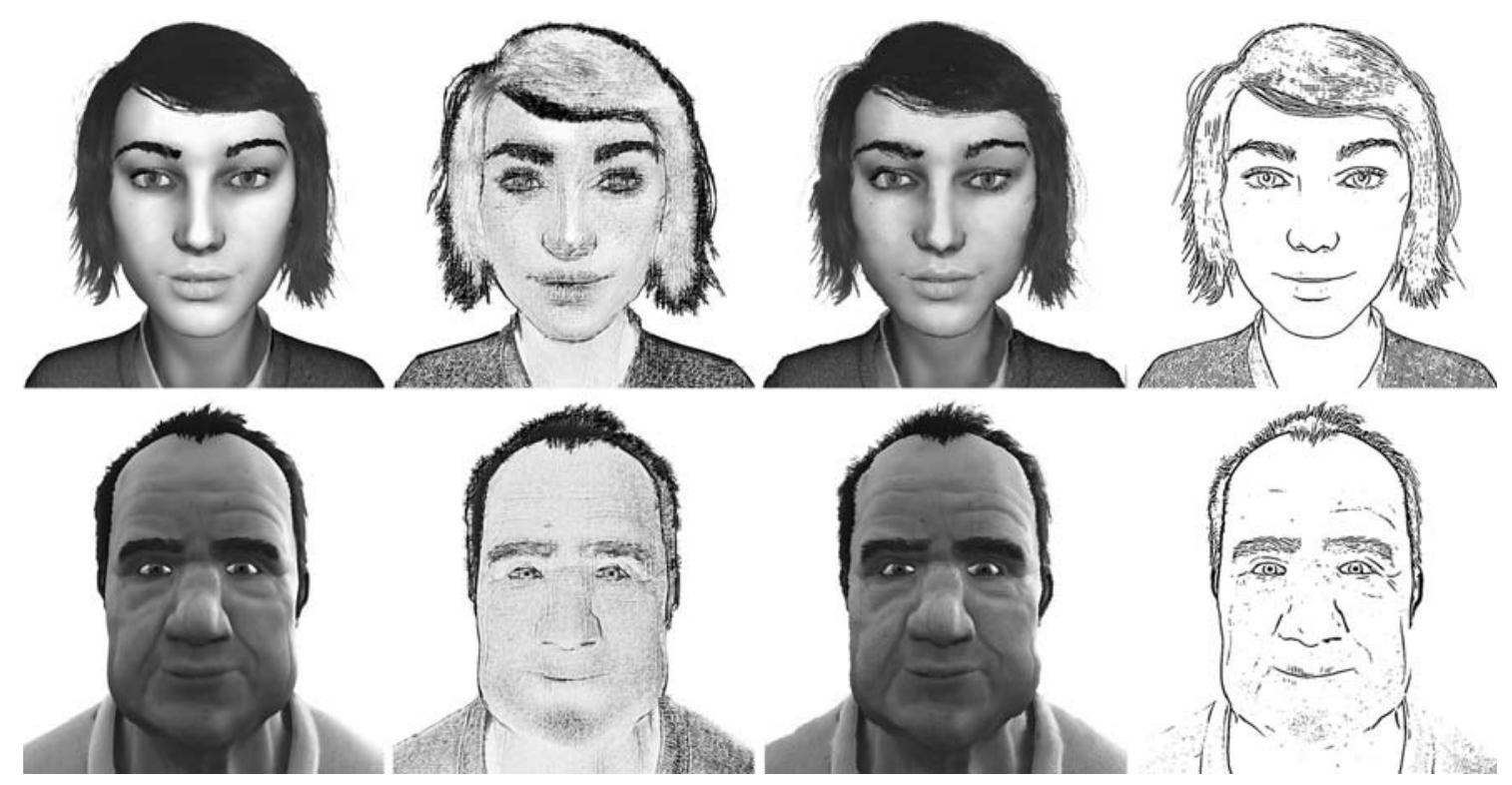

Fig. 4 NPR styles (medium abstraction level) applied to Nikita (upper row) and Hank (lower row). Left to right image abstraction, pencil drawing, watercolor, coherent line drawing

- Coherent line drawing $(C L D)$;

- Pencil drawing $(P D)$;

- Image abstraction (IA) ;

- Watercolors $(W)$.

These were instantiated in one of three levels of abstraction, i.e., low, medium and high.

\subsection{Procedure}

The way each session was carried out is similar to the R-DECT. The NPR-DECT had a duration of approximately $20 \mathrm{~min}$. At the end of each session, the participants needed to fill a computer-based questionnaire to rate the likability and recognizability of the abstracted faces using NPR styles.

\subsubsection{Likeability and recognizability questionnaire}

The questionnaire comprised 26 images: 2 (characters) $\times 4$ (stylized faces by NPR) $\times 3$ (levels of abstraction) +2 (realistic-looking Hank and realistic-looking Nikita). For each image, two questions were posed:

1. How good were you able to recognize the emotions from this representation?

2. How did you like this representation?

For each question, the answers were presented in a 7-point Likert scale ranging from very good ("1") to very bad ("7").

Additionally, during the whole NPR-DECT session participants' gaze was tracked by an RED-250 eye tracker
(SMI). The eye tracker was integrated in Frapper to allow the communication between each other.

\subsection{Results}

In total, $71.4 \%$ of the emotional expressions were correctly categorized. Due to technical problems in one session, rating data are based on $n=30$ participants only.

We found a considerable difference of accuracy between both characters: Hank: 66.5\% versus Nikita 76.3\% (Fig. 5).

Differences in accuracy levels seem to be dependent on the character in a certain style with a certain level of abstraction. For Hank, the lowest rate was obtained with CLD - high abstraction (55.9\%), whereas the highest rate was with $W-$ medium abstraction $(73.7 \%)$. For Nikita, the lowest percentage was obtained with $P D$-medium abstraction (71.5\%), whereas the highest percentage was noted for $I A$ - low abstraction ( $81.7 \%$ ).

Regarding the recognizability and likability rates (Table 1), these were the highest for the original representation $(M=2.02, \mathrm{SD}=1.11), \quad$ followed by $I A$ $(M=2.44, \mathrm{SD}=1.05), C L D(M=3.69, \mathrm{SD}=1.70), W$ $(M=3.73, \mathrm{SD}=1.39), \quad$ and finally $P D \quad(M=4.17$, $\mathrm{SD}=1.33)$.

\section{Experiment 3: Gaze Behavior}

The current experiment addressed the question whether deviant gaze behavior and higher scores of alexithymia are more common in individuals with ASD when compared to 


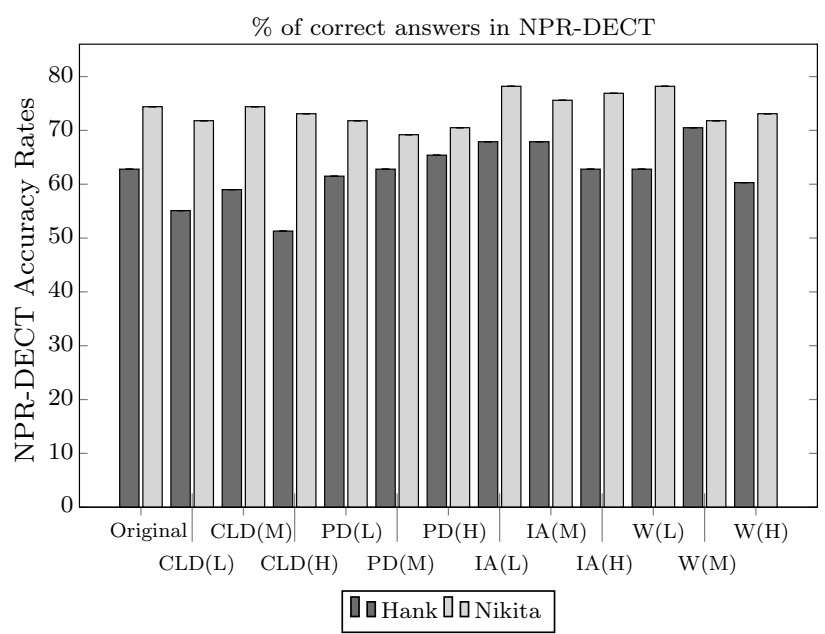

Fig. 5 Percentage (\%) of correct answers in NPR-DECT. CLD Line drawing, $P D$ pencil drawing, $W$ watercolor, $I A$ image abstraction, $L$ low abstraction, $M$ medium abstraction, $H$ high abstraction

NTD controls. Alexithymia describes patients' difficulties in communicating due to the "inability to find appropriate words to describe their feelings" [36].

Our study is based on the work of Bird et al. [22], where they proposed a possible solution for the ongoing debate about gaze behavior and ASD. They conducted an eyetracking study with adults with and without autism. Bird et al. interpret their results as it is the degree of alexithymia that influences a person's eye fixation rather than the severity degree of autistic symptomatology. This means that individuals with autism only display anomalous eye contact when they suffer from comorbid alexithymia.

The novelty of our work is to investigate whether Bird et al.'s findings in an adult sample could be replicated using an adolescent sample. We also attempt to improve their scientific and methodological approach. This study represents another use case of the multimodal potential of the SARA testbed.

\subsection{Participants}

Our sample consisted of 35 male adolescents aged 14 and 17 years. From this group, 14 were diagnosed with ASD and 21 were NTD. The mean age in the ASD group was 15.33 years $(\mathrm{SD}=0.99)$ and in the NTD group 15.64 years $(\mathrm{SD}=1.15)$.

The mean IQ, measured by the CFT 20-R [37], was $103.71(\mathrm{SD}=14.31)$ for the ASD group and 103.48 $(\mathrm{SD}=8.94)$ for NTDs.

Individuals with ASD who also met the criteria for ADHD were not excluded from the study. Apart from that, participants who needed a correction to the visual acuity higher than 1.5 diopters and therefore need to wear glasses or soft contact lenses, were excluded since the eye tracking
Table 1 Mean of (a) recognizability and (b) likeability ratings of the rendering styles and virtual characters (VC) from 1 (very good) to 7 (very bad)

\begin{tabular}{|c|c|c|c|c|}
\hline Style & $M(H)$ & $\mathrm{SD}(H)$ & $M(N)$ & $\mathrm{SD}(N)$ \\
\hline \multicolumn{5}{|c|}{ (a) Recognizability } \\
\hline Ori & 2.27 & 1.34 & 1.77 & 0.77 \\
\hline CLD (L) & 3.67 & 1.35 & 3.07 & 1.6 \\
\hline CLD (M) & 3.83 & 1.64 & 3.07 & 1.74 \\
\hline CLD $(\mathrm{H})$ & 4.73 & 1.74 & 3.80 & 1.67 \\
\hline $\mathrm{PD}(\mathrm{L})$ & 4.53 & 1.28 & 3.80 & 1.24 \\
\hline $\mathrm{PD}(\mathrm{M})$ & 4.00 & 1.14 & 4.07 & 1.55 \\
\hline $\mathrm{PD}(\mathrm{H})$ & 4.27 & 1.23 & 4.37 & 1.45 \\
\hline IA (L) & 2.10 & 1.03 & 1.77 & 0.5 \\
\hline IA (M) & 2.50 & 0.97 & 2.10 & 0.76 \\
\hline IA $(\mathrm{H})$ & 3.10 & 1.12 & 3.07 & 1.05 \\
\hline W (L) & 3.53 & 1.36 & 3.00 & 1.23 \\
\hline W (M) & 3.67 & 1.30 & 3.17 & 1.23 \\
\hline $\mathrm{W}(\mathrm{H})$ & 4.63 & 1.22 & 4.37 & 1.33 \\
\hline \multicolumn{5}{|c|}{ (b) Likeability } \\
\hline Ori & 2.70 & 1.18 & 2.07 & 1.14 \\
\hline CLD (L) & 4.00 & 1.29 & 3.23 & 1.38 \\
\hline CLD (M) & 4.07 & 1.31 & 3.20 & 1.47 \\
\hline CLD (H) & 4.83 & 1.39 & 3.90 & 1.49 \\
\hline $\mathrm{PD}(\mathrm{L})$ & 4.47 & 1.22 & 4.70 & 1.37 \\
\hline PD (M) & 4.20 & 1.27 & 4.37 & 1.67 \\
\hline $\mathrm{PD}(\mathrm{H})$ & 4.20 & 1.21 & 4.93 & 1.46 \\
\hline IA (L) & 2.57 & 1.17 & 2.03 & 0.81 \\
\hline IA (M) & 2.90 & 1.16 & 2.47 & 1.22 \\
\hline IA $(\mathrm{H})$ & 3.33 & 1.37 & 3.60 & 1.33 \\
\hline W (L) & 3.87 & 1.43 & 3.60 & 1.43 \\
\hline W (M) & 4.20 & 1.37 & 3.77 & 1.38 \\
\hline $\mathrm{W}(\mathrm{H})$ & 4.90 & 1.32 & 4.87 & 1.38 \\
\hline
\end{tabular}

Mean values are color-coded from white to red, more intense red meaning worse

$M(H)$ : Hank's mean values, SD $(H)$ standard deviation of Hank's mean values, $M(N)$ Hank's mean values, $\mathrm{SD}(N)$ standard deviation of Nikita's mean values

would be inaccurate for higher degrees of diopters. This however was not necessary for participants who wore rigid gas-permeable lenses. Additionally, former participation in any experiments with one of the DECT versions was one of the exclusion criteria of the study.

\subsection{Stimuli}

The stimuli for this study corresponded to the NPR-DECT. As such, the test presented: 2 (characters) $\times 6$ (basic emotions $) \times 13(1$ original style +12 NPR style $\times$ abstraction combinations) $=156$ trials.

However, owing to the amount of data to be evaluated, in this paper we present some preliminary results that 
correspond to the evaluation of only 12 animations of the characters in the realistic-looking style $(2$ characters $\times 6$ basic emotions).

\subsection{Procedure}

The participants underwent other tasks that included: emotional categorization, assessment of their IQ and different questionnaires. The whole experimental session took approximately $90 \mathrm{~min}$ per participant.

To collect gaze behavior data during emotional categorization, we used the RED250 remote eye-tracking system (SensoMotoric Instruments-SMI, Teltow, Germany). Among its advantages are its ease of use, high data quality with high-speed capabilities, and calibration times, which are of the order of few seconds. Moreover, it offers the possibility to work with subjects that wear most glasses and contact lenses (these are specified by the manufacturer). Nevertheless, for the present study the participants either wore no glasses or wore rigid contact lenses.

For our study, it was necessary to communicate Frapper with the eye tracker. To that end, we implemented a plugin in Frapper that established a network communication between the test (R-DECT or NPR-DECT) and the eye tracker SDK.

The procedure to perform the eye tracking required the participant to seat in front of the RED250 and computer screen. Then, chair, chin rest, table and monitor were properly adjusted according to the person's height. Subsequently, the calibration was conducted and the actual experiment began. The participant was asked to move as little as possible in order to minimize artifacts in the eyetracking data.

The emotional categorization was carried out using the NPR-DECT, which took approximately 15-20 min. The answers to the categorization were typed in by the experimenter to avoid distortions in the eye-tracking data.

Additionally, a number of instruments were used to measure intelligence quotient and degree of alexithymia (Table 2).

\subsubsection{Dependent variables}

Three dependent variables were considered: gaze behavior, correctness of the answers and reaction times.

The gaze behavior was assessed by considering the "total dwell time" (TDT), which is the sum of sample durations for all gaze data samples that hit the area of interest (AOI) [38]. The relevant AOIs were eyes, mouth and face (Fig. 6).

The TDTs on these specific AOIs were further used to calculate face-non-face difference (FNFD) and eye-mouth difference (EMD). According to Bird et al. [22], FNFD serves as an index of social attention. EMD is the total fixation time on the eyes compared to the mouth.

A value of 0 indicates equal attention to facial and nonfacial stimuli, as well as equal fixations on the eyes and mouth. Values greater than 0 imply attentional preference toward facial stimuli (or the eyes) compared to non-facial stimuli (or the mouth). Values lower than 0 signify the opposite case.

The methodological difference between our study and that of Bird et al. is that they used ratios between face-nonface fixations and mouth-eyes fixations, whereas the present study uses differences because there were cases where the denominator value was 0 , causing division by zero.

The second variable, correctness of answers, regarded the 12 relevant NPR-DECT items.

The third dependent measure, reaction times, was obtained through the data recording program PsychoPy in combination with Frapper.

\subsubsection{Hypotheses}

The research consisted of a quasi-experimental between subjects design. We considered as independent variable group affiliation (ASD vs. NTD), as dependent variable gaze behavior (FNFD and EMD), and as presumed moderator variable degree of alexithymia. The latter was measured via TAS-26 [39] and BVAQ-AB [40]. The data collected was preprocessed using the eye-tracking analysis program SMI BeGaze, Version 3.5.

Three hypothesis were formulated:
Table 2 Overview and description of instruments used in the gaze behavior study

\begin{tabular}{llll}
\hline Instrument & Performer & Type of instrument & Approximate duration (min) \\
\hline NPR-DECT & Participant & Computer test (ET) & 20 \\
CFT 20-R & Participant & Paper-pencil IQ test & 30 \\
TAS-26 & Participant & Paper-pencil questionnaire & 10 \\
BVAQ-AB & Participant & Paper-pencil questionnaire & 10 \\
\hline
\end{tabular}

NPR-DECT Non-Photorealistic Rendering Dynamic Emotion Categorization Test, CFT 20-R Culture Fair Intelligence Test, TAS-26 Toronto Alexithymia Scale, BVAQ-AB Bermond-Vorst Alexithymia Questionnaire 

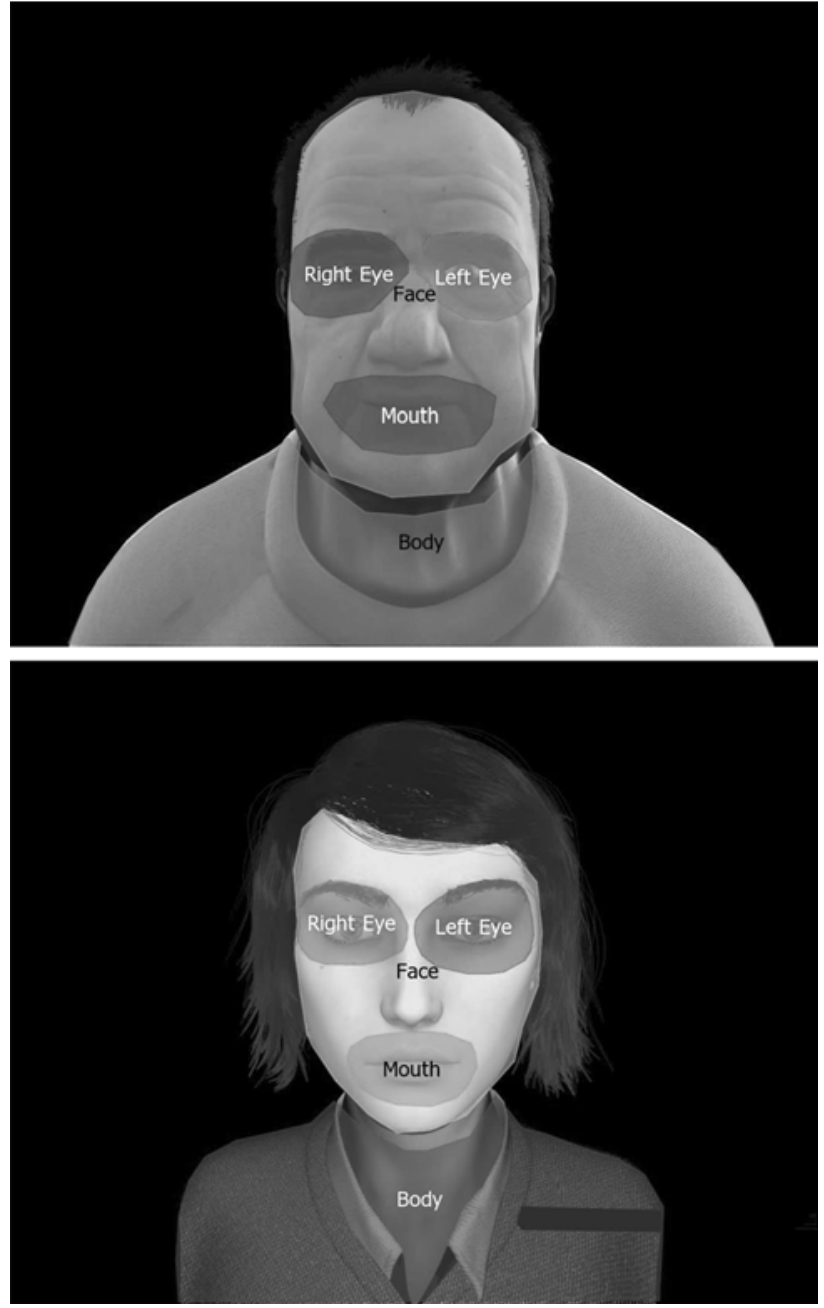

Fig. 6 Examples of stimuli with defined AOIs

- H1: The group affiliation will influence gaze behavior. In detail, control participants (NTDs) will fixate social stimuli longer and more frequently than ASDs;

- H2: The higher a participant's alexithymia score, the less frequent and shorter social relevant stimuli will be fixated (i.e., lower FNFD and EMD difference);

- H3: The group affiliation influences the degree of alexithymia. In detail, ASDs will score higher on the alexithymia instruments than NTDs.

\subsubsection{Measures}

To test the first hypothesis, we conducted a one factorial ANOVA with the independent variable group affiliation (ASD vs. NTD) and the dependent variable gaze behavior (FNFD and EMD).

For the second hypothesis, we computed Spearman correlations regarding gaze behavior, and mean scores of TAS-26 and BVAQ-AB.

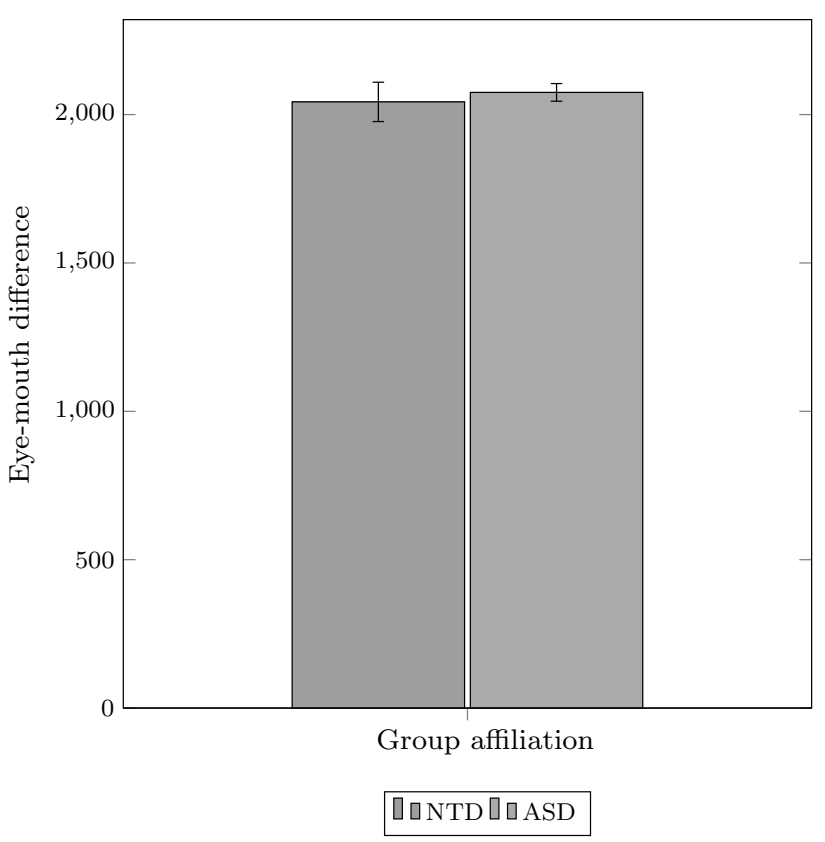

Fig. 7 Group difference regarding the FNFD of AOI-based total dwell times. Error bars represent 95\% CI

As for the third hypothesis, we calculated another one factorial ANOVA with the independent variable group affiliation (ASD vs. NTD) and the variable degree of alexithymia.

\subsection{Results}

A one-way ANOVA on age and IQ revealed no significant group difference in terms of age $(F(1,33)<1)$, or as to the IQ $(F(1,33)<1)$.

\subsubsection{Hypothesis 1}

We tested whether the group affiliation (ASD vs. NTD) would influence a person's gaze behavior. Regarding the FNFD, a Mann-Whitney U test was used to check a significant effect of group affiliation on this gaze behavior. In this respect, no significant main effect was found $(U=139.00, z=-0.27, p=0.803)$. This implies that there were no larger differences between ASDs $(M=$ 2074.87, SD $=58.82)$ and NTDs $(M=2042.88, \mathrm{SD}=$ 154.99) (Fig. 7). When observing the data on a descriptive level, one can notice that NTDs actually showed lower FNFD values than ASDs, which is contrary to hypothesis 1.

As for EMD, the ANOVA revealed no significant group effect either. This means that ASDs $(M=407.98, \mathrm{SD}=$ 766.26) did not differ significantly from NTDs $(M=261.93, \mathrm{SD}=817.16), F(1,34)<1$ (Fig. 8). Once again these findings demonstrate a direction contrary to our hypothesis on a descriptive level indicating that NTDs had lower EMD scores than ASDs. 


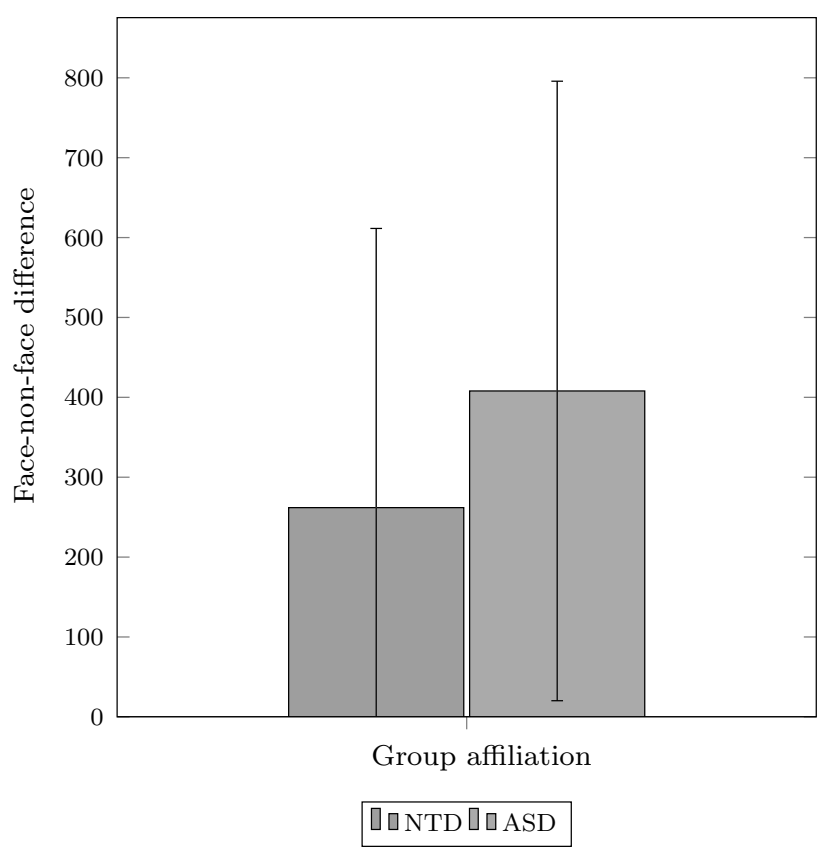

Fig. 8 Group difference regarding the EMD of AOI-based total dwell times. Error bars represent $95 \%$ CI

\subsubsection{Hypothesis 2}

To test whether the degree of alexithymia has a direct impact on the participant's gaze behavior, we used Pearson correlations. The analysis was carried out on both the total sample and within groups.

Contrary to the hypothesis, none of the correlations between the alexithymia instruments and the FNFD value were significant, neither for the total sample nor within both groups regarded separately (Tables 3, 4, 5).

One significant moderate relationship between the EOT (externally oriented thinking) scale of the TAS-26 and the EMD value $(r=0.35, p=0.046)$ appeared in the total sample (Table 6). When observing this relationship within groups, it still remained significant for the NTDs
( $r=0.50, p=0.024)$, whereas there was no significant relationship within the ASD subsample (Tables 7, 8). When considering NTDs only, we also found a moderate correlation between the verbalizing scale of the BVAQ-AB and the EMD value. However, this effect was only a statistical tendency $(r=0.42, p=0.067)$. All of the other correlations between the alexithymia instruments and the EMD score were nonsignificant.

\subsubsection{Hypothesis 3}

This hypothesis states that the group membership influences the degree of alexithymia. To test this, two separate analyses of variance for the TAS-26 and BVAQ-AB were performed.

A Shapiro-Wilk test, concerning TAS-26 scores, revealed that in both groups the normality assumption was not violated, with the exception of the DIF (difficulty identifying feelings) scale: NTD $(W=0.90, p=0.046)$, and the ASD $(W=0.86, p=0.033)$. Therefore, a MannWhitney U test was applied for this scale, whereas ANOVAs were used for the remaining scales.

For the overall TAS-26 scale, a significant main effect was revealed, $F(1,32)=5.14, p=0.030, g=0.95$. It implies that ASDs scored higher $(M=43.79, \mathrm{SD}=9.78)$ than NTD participants $(M=37.15, \mathrm{SD}=7.32)$ (Fig. 9).

Another significant difference was found for the DIF (difficulty identifying feelings) and DDF (difficulty describing feelings) scales combined, $F(1,32)=5.29$, $p=0.028, g=1.02$, where participants with ASD scored significantly higher $(M=28.21, \mathrm{SD}=10.14)$ than their NTD peers $(M=21.90, \mathrm{SD}=5.85)$.

Additionally, we found a marginally significant difference between ASDs and NTDs concerning the DDF scale, $F(1,32)=3.02, p=0.092, g=0.59$, indicating once again higher values for ASDs $(M=14.14, \mathrm{SD}=5.20)$ than for NTDs $(M=11.40, \mathrm{SD}=4.01)$. See Table 9 for an overview.
Table 3 Correlations between the TAS-26 with respect to the BVAQ-AB and the FNFD for the total sample

\begin{tabular}{cllcccc}
\hline \multicolumn{7}{c}{ Correlations between TAS-26 and FNFD $(n=34)$} \\
\cline { 2 - 7 } & \multicolumn{1}{l}{ Overall score } & DIF & DDF & EOT & DIF + DDF \\
\hline FNFD & $r=-0.08$ & $r=0.15$ & $r=-0.15$ & $r=-0.20$ & $r=0.002$ \\
& $p=0.648$ & $p=0.394$ & $p=0.389$ & $p=0.251$ & $p=0.993$ \\
\hline \multirow{6}{*}{ Correlations between BVAQ-AB and FNFD $(n=34)$} & & & \\
\cline { 2 - 7 } & Overall score & Emotionalizing & Verbalizing & Fantasizing & Identifying & Analyzing \\
\hline FNFD & $r=-0.13$ & $r=-0.18$ & $r=-0.02$ & $r=0.05$ & $r=-0.05$ & $r=-0.24$ \\
& $p=0.484$ & $p=0.309$ & $p=0.921$ & $p=0.764$ & $p=0.777$ & $p=0.182$ \\
\hline
\end{tabular}

$T A S$-26 Toronto Alexithymia Scale, $B V A Q-A B$ Bermond-Vorst Alexithymia Scale, $F N F D$ face-non-face difference, $D I F$ difficulty identifying feelings, $D D F$ difficulty describing feelings to others, $E O T$ externally oriented thinking 
Table 4 Correlations between the TAS-26 with respect to the BVAQ-AB and the FNFD for ASDs

Table 5 Correlations between the TAS-26 with respect to the BVAQ-AB and the FNFD for NTDs

Table 6 Correlations between the TAS-26 and BVAQ-AB with respect to the EMD for the total sample

\begin{tabular}{llllll}
\hline & \multicolumn{4}{l}{ Correlations between TAS-26 and FNFD $(n=14)$} \\
\cline { 2 - 6 } & Overall score & DIF & DDF & EOT & DIF + DDF \\
\hline FNFD & $r=-0.09$ & $r=-0.21$ & $r=0.20$ & $r=-0.22$ & $r=-0.02$ \\
& $p=0.749$ & $p=0.466$ & $p=0.488$ & $p=0.458$ & $p=0.939$ \\
\hline
\end{tabular}

Correlations between BVAQ-AB and FNFD $(n=14)$

\begin{tabular}{lllllll} 
& $\begin{array}{l}\text { Overall } \\
\text { score }\end{array}$ & Emotionalizing & Verbalizing & Fantasizing & Identifying & Analyzing \\
\hline FNFD & $r=-0.25$ & $r=-0.17$ & $r=-0.01$ & $r=-0.19$ & $r=-0.08$ & $r=-0.26$ \\
& $p=0.399$ & $p=0.570$ & $p=0.964$ & $p=0.514$ & $p=0.780$ & $p=0.363$ \\
\hline
\end{tabular}

$T A S-26$ Toronto Alexithymia Scale, $B V A Q-A B$ Bermond-Vorst Alexithymia Scale, $F N F D$ face-non-face difference, $A S D$ autism spectrum disorder, $D I F$ difficulty identifying feelings, $D D F$ difficulty describing feelings to others, EOT externally oriented thinking

\begin{tabular}{llllll}
\hline \multicolumn{7}{l}{ Correlations between TAS-26 and FNFD $(n=21)$} \\
\cline { 2 - 6 } & Overall score & DIF & DDF & EOT & DIF + DDF \\
\hline FNFD & $r=-0.18$ & $r=0.34$ & $r=-0.37$ & $r=-0.22$ & $r=-0.07$ \\
& $p=0.458$ & $p=0.141$ & $p=0.110$ & $p=0.355$ & $p=0.765$ \\
\hline
\end{tabular}

Correlations between BVAQ-AB and FNFD $(n=21)$

\begin{tabular}{lllllll} 
& $\begin{array}{lllll}\text { Overall } \\
\text { score }\end{array}$ & Emotionalizing & Verbalizing & Fantasizing & Identifying & Analyzing \\
\hline FNFD & $r=-0.15$ & $r=-0.22$ & $r=-0.05$ & $r=0.18$ & $r=-0.13$ & $r=-0.31$ \\
& $p=0.533$ & $p=0.347$ & $p=0.851$ & $p=0.453$ & $p=0.588$ & $p=0.186$ \\
\hline
\end{tabular}

$T A S$-26 Toronto Alexithymia Scale, $B V A Q-A B$ Bermond-Vorst Alexithymia Scale, $F N F D$ face-non-face difference, $A S D$ autism spectrum disorder, $D I F$ difficulty identifying feelings, $D D F$ difficulty describing feelings to others, EOT externally oriented thinking

\begin{tabular}{llllll}
\hline & \multicolumn{6}{l}{ Correlations between TAS-26 and EMD $(n=34)$} \\
\cline { 2 - 6 } & Overall score & DIF & DDF & EOT & DIF+DDF \\
\hline EMD & $r=0.31$ & $r=0.16$ & $r=0.15$ & $r=0.35$ & $r=0.18$ \\
& $p=0.078$ & $p=0.379$ & $p=0.385$ & $p=0.046$ & $p=0.318$ \\
\hline
\end{tabular}

Correlations between BVAQ-AB and $\operatorname{EMD}(n=14)$

\begin{tabular}{lllllll} 
& $\begin{array}{lllll}\text { Overall } \\
\text { score }\end{array}$ & Emotionalizing & Verbalizing & Fantasizing & Identifying & Analyzing \\
\hline FNFD & $r=0.27$ & $r=-0.15$ & $r=0.24$ & $r=0.19$ & $r=0.11$ & $r=0.22$ \\
& $p=0.118$ & $p=0.398$ & $p=0.172$ & $p=0.277$ & $p=0.531$ & $p=0.214$ \\
\hline
\end{tabular}

TAS-26 Toronto Alexithymia Scale, $B V A Q-A B$ Bermond-Vorst Alexithymia Scale, EMD eye-mouth difference, $D I F$ difficulty identifying feelings, $D D F$ difficulty describing feelings to others, $E O T$ externally oriented thinking
On the contrary, the group difference with respect to the overall BVAQ-AB scale was not significant $(F(1,32)<1)$, and neither were group differences in terms of the subscales.
However, we were able to reveal one marginally significant main effect, as for the subscale DIF, $F(1,32)=3.14, p=0.086$, meaning that NTDs $(M=$ $15.55, \mathrm{SD}=4.95)$ exhibit less problems when it comes to 
Table 7 Correlations between the TAS-26 and BVAQ-AB with respect to the EMD for ASDs

Table 8 Correlations between the TAS-26 and BVAQ-AB with respect to the EMD for NTDs

\begin{tabular}{llllll}
\hline & \multicolumn{4}{l}{ Correlations between TAS-26 and EMD $(n=14)$} \\
\cline { 2 - 6 } & Overall score & DIF & DDF & EOT & DIF+DDF \\
\hline EMD & $r=0.23$ & $r=0.27$ & $r=0.12$ & $r=-0.03$ & $r=0.23$ \\
& $p=0.439$ & $p=0.344$ & $p=0.677$ & $p=0.930$ & $p=0.438$ \\
\hline
\end{tabular}

Correlations between BVAQ-AB and EMD $(n=14)$

\begin{tabular}{lllllll} 
& $\begin{array}{l}\text { Overall } \\
\text { score }\end{array}$ & Emotionalizing & Verbalizing & Fantasizing & Identifying & Analyzing \\
\hline FNFD & $r=0.17$ & $r=-0.28$ & $r=-0.01$ & $r=0.20$ & $r=0.25$ & $r=0.09$ \\
& $p=0.559$ & $p=0.334$ & $p=0.975$ & $p=0.483$ & $p=0.385$ & $p=0.751$ \\
\hline
\end{tabular}

TAS-26 Toronto Alexithymia Scale, $B V A Q-A B$ Bermond-Vorst Alexithymia Scale, $E M D$ eye-mouth difference, $A S D$ autism spectrum disorder, $D I F$ difficulty identifying feelings, $D D F$ difficulty describing feelings to others, EOT externally oriented thinking

\begin{tabular}{llllll}
\hline & \multicolumn{5}{l}{ Correlations between TAS-26 and EMD $(n=21)$} \\
\cline { 2 - 6 } & Overall score & DIF & DDF & EOT & DIF+DDF \\
\hline EMD & $r=0.31$ & $r=-0.06$ & $r=0.10$ & $r=0.50^{*}$ & $r=0.04$ \\
& $p=0.191$ & $p=0.804$ & $p=0.666$ & $p=0.024$ & $p=0.871$ \\
\hline
\end{tabular}

Correlations between BVAQ-AB and EMD $(n=21)$

\begin{tabular}{lllllll} 
& $\begin{array}{l}\text { Overall } \\
\text { score }\end{array}$ & Emotionalizing & Verbalizing & Fantasizing & Identifying & Analyzing \\
\hline FNFD & $r=0.35$ & $r=-0.07$ & $r=0.42^{+}$ & $r=0.18$ & $r=-0.13$ & $r=-0.31$ \\
& $p=0.133$ & $p=0.769$ & $p=0.067$ & $p=0.453$ & $p=0.588$ & $p=0.186$ \\
\hline
\end{tabular}

$T A S$-26 Toronto Alexithymia Scale, $B V A Q-A B$ Bermond-Vorst Alexithymia Scale, EMD eye-mouth difference, $A S D$ autism spectrum disorder, $D I F$ difficulty identifying feelings, $D D F$ difficulty describing feelings to others, $E O T$ externally oriented thinking.

${ }^{+} p<0.10, * p<0.05, * * p<0.01, * * * p<0.001$

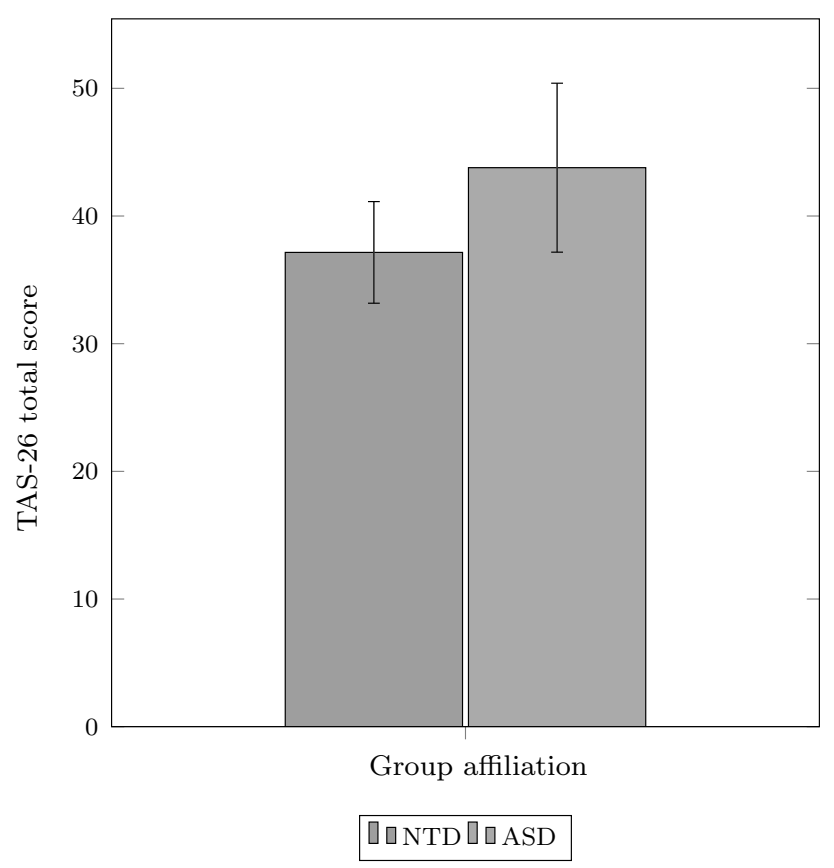

Fig. 9 Group differences on the total TAS-26 score the identification of emotions than ASDs $(M=$ 19.79, $\mathrm{SD}=8.95)$ do (Fig. 10; Table 10).

The analysis of the correlation between recognition of emotions and overall scales and subscales for the assessment of alexithymia (TAS-26 and BVAQ-AB) showed that there were no significant correlations.

Conversely, the correlation between the total scores of both instruments (TAS-26 and BVAQ-AB) is a strong one $(r=0.69, p<0.001)$.

\section{Discussion}

\subsection{R-DECT}

The results of the experiment validated the R-DECT (no floor effects or ceiling effects appeared), proving that it can be used to assess the general ability of categorizing facial expressions of emotions. Moreover, the test was able to detect the impairments in facial emotion categorization in 
Table 9 Group differences on the total score and subscales of the TAS-26

\begin{tabular}{|c|c|c|c|c|c|c|c|c|}
\hline \multirow[t]{2}{*}{ TAS-26 Scale } & \multicolumn{2}{|c|}{$\operatorname{ASD}(n=14)$} & \multicolumn{2}{|c|}{$\operatorname{NTD}(n=21)$} & \multicolumn{4}{|c|}{ Statistics } \\
\hline & $M$ & SD & $M$ & SD & $F$ & $p$ & $g$ & \\
\hline Overall scale & 43.79 & 6.03 & 37.15 & 7.32 & $5.14^{*}$ & 0.030 & 0.95 & \\
\hline DDF & 14.14 & 5.20 & 11.40 & 4.01 & $3.02^{+}$ & 0.092 & 0.59 & \\
\hline EOT & 15.57 & 3.20 & 15.25 & 3.99 & 0.06 & 0.804 & 0.08 & \\
\hline $\mathrm{DIF}+\mathrm{DDF}$ & 28.21 & 6.27 & 21.90 & 5.85 & $5.29^{*}$ & 0.028 & 1.02 & \\
\hline & & & & & $U$ & $z$ & $p$ & $g$ \\
\hline DIF & 14.07 & 6.03 & 10.50 & 3.10 & 94.00 & -1.61 & 0.106 & 0.78 \\
\hline
\end{tabular}

TAS-26 Toronto Alexithymia Scale, ASD autism spectrum disorder, NTD neurotypically developed, DIF difficulty identifying feelings, $D D F$ difficulty describing feelings to others, EOT externally oriented thinking, $g$ Hedge's $g$

${ }^{+} p<0.10, * p<0.05, * * p<0.01, * * * p<0.001$

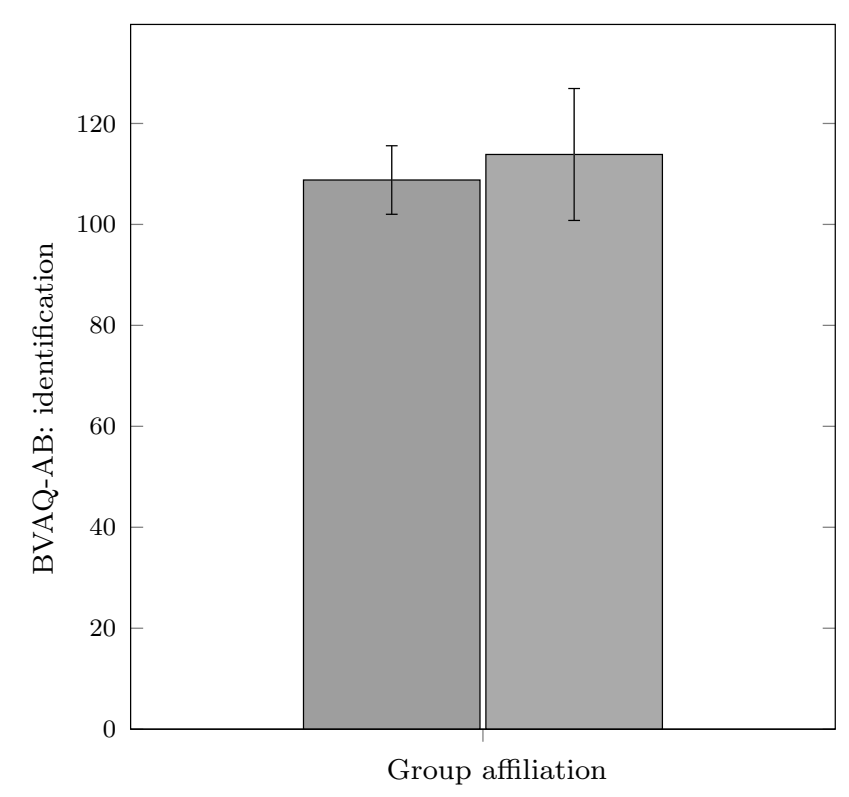

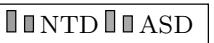

Fig. 10 Group differences on the BVAQ-AB subscale "identifying feelings"

the ASD group. However, the statistical power of the study was too low to assess which emotion(s) contributed to this general difference.

The R-DECT also served to validate the generated facial animations, shedding light on the improvements that needed to be done to convey the corresponding emotional meaning. The intensity of the displayed emotional facial expressions was shown to play a considerable role in categorization. To improve the test, some adjustments of animations had to be made especially in the condition where intensity of the expression was intended to be "medium" or "strong." That seems to be the case especially for the animation expressing the emotion fear.

\subsection{NPR-DECT}

The preliminary results of the NPR-DECT, considering only a sample of neurotypically developed (NTD) participants, gave some insight on the categorization and perception of the stylization methods used to abstract the characters' facial expressions.

As for recognizability and likeability, it could be observed that, apart from the realistic-looking (original) style, the "image abstraction" stylization in all levels led to better rates in both characters. Regarding the other styles, we could not draw any definite conclusion given that no clear recognition or likeability pattern arose.

We also found a significant difference of accuracy between both virtual characters, being Nikita the one that facilitated the categorization of emotions across all NPR styles.

\subsection{Gaze behavior}

The results of the experiment regarding the first hypothesis did not replicate anomalies on the gaze variables FNFD (face-non-face difference) and EMD (eye-mouth difference). In fact, the conducted analysis revealed no differences between both the ASD and NTD groups.

Some arguments that might justify this result include differences in the stimuli in our experiments, compared to the stimuli in Bird et al. [22] (we used animated characters and they employed footage with real actors); duration of the trials (theirs were considerably longer); and the presence of distracting content (in Bird et al.'s study, participants could choose not to look at the characters, while in our test the characters occupied most of the screen space). Another possible explanation might be a considerable number of ASD participants that took part in another study of our department about five years ago. On the one hand, this speaks in favor of an especially motivated sample. On the other hand, it also signifies that these patients received their diagnoses in early childhood and had the chance to work on their deficits. Finally, it might be that there is indeed 
Table 10 Group differences on the total score and subscales of the BVAQ-AB

\begin{tabular}{|c|c|c|c|c|c|c|c|}
\hline \multirow[t]{2}{*}{ BVAQ-AB Scale } & \multicolumn{2}{|c|}{$\operatorname{ASD}(n=14)$} & \multicolumn{2}{|c|}{$\operatorname{NTD}(n=21)$} & \multicolumn{3}{|c|}{ Statistics } \\
\hline & $M$ & SD & $M$ & SD & $F$ & $p$ & $g$ \\
\hline Overall scale & 113.86 & 19.31 & 108.80 & 12.48 & 0.79 & 0.380 & 0.32 \\
\hline Emotionalizing & 24.27 & 4.20 & 24.30 & 3.23 & 0.00 & 0.978 & 0.01 \\
\hline Verbalizing & 24.50 & 7.59 & 23.06 & 5.41 & 0.42 & 0.521 & 0.22 \\
\hline Fantasizing & 22.14 & 8.71 & 24.49 & 6.57 & 0.81 & 0.376 & 0.31 \\
\hline Identifying & 19.79 & 8.95 & 15.55 & 4.95 & $3.14^{+}$ & 0.086 & 0.61 \\
\hline Analyzing & 23.64 & 6.27 & 22.10 & 4.67 & 0.68 & 0.416 & 0.28 \\
\hline
\end{tabular}

$B V A Q-A B$ Bermond-Vorst Alexithymia Scale, ASD autism spectrum disorder, $N T D$ neurotypically developed, $g$ Hedge's g

${ }^{+} p<0.10, * p<0.05, * * p<0.01, * * * p<0.001$ no connection between high-functioning ASD and deviant gaze behavior, as concluded by Sawyer et al. [21].

The findings concerning the second hypothesis indicated that alexithymia has only little to no influence on the gaze behavior (FNFD or EMD). One exception on the overall results was the EOT (externally oriented thinking) subscale from the TAS-26, where there was a moderate relationship between EOT and EMD. This correlation became stronger regarding only the NTD group, though it was not significant within the ASD group. A reason for this could be that EOT measures a construct different than the other two scales, DIF (difficulty identifying feelings) and DDF (difficulty describing feelings to others), or the overall scale.

Finally, the results partially support the assumption that ASDs show higher values of alexithymia. In detail, there was strong evidence for this hypothesis when considering the overall TAS-26 scale and the scales DIF and DDF combined. However, there were no significant group differences when utilizing the BVAQ-AB. One could argue that the TAS-26 scale has been a widely used instrument for the assessment of alexithymia, including adolescent samples from the age of 14 and above. On the contrary, the $\mathrm{BVAQ}-\mathrm{AB}$ is a relatively new instrument that has not been validated for adolescent populations yet. Hence, it might be that the BVAQ-AB is only validly applicable on adults. One has to notice that the BVAQ-AB comprises 40 items as compared to only 26 TAS-26 items. Considering that the participants had to complete four questionnaires prior to the experiment, a longer questionnaire like the BVAQ-AB might have resulted in minimized motivation rates.

Further limitations that might have contributed to the noncorroboration of the hypotheses are: the small overall sample $(n=35)$; ADHD comorbidity in the study; the use of the eyetracking variable FNFD (face-non-face difference), which does not entirely fit into our research because the faces of the characters occupy most of the screen, as well as the time given to look at the stimuli (less than $2 \mathrm{~s}$ ); or possible influences of training and psychotherapy on gaze behavior along the years.

\section{Conclusions}

In this paper, we have compiled the results of previous experiments carried out within the SARA project. In particular, the focus was emotion categorization of facial expressions, and the relation between alexithymia, ASD and eye gaze.

As for the emotional categorization, we validated the use of animated facial expressions to work in autism research. They provide the flexibility and parameterization required in an experiment, which could be personalized according to the participants' or experimenters' needs.

We also introduced a novel element, i.e., stylization of facial expressions. The validation of the different abstracted representations was assessed by NTD participants. However, the most important conclusion we could draw was that styles resembling the original one (e.g., image abstraction) were preferred. Nevertheless, we could not assess the differences between styles and what elements should be improved.

Regarding gaze behavior and its link to alexithymia and ASD, our results did not reproduce the ones from Bird et al. [22]. This might indicate that there is indeed a relationship between gaze behavior and ASD, which will also be explored in our future research.

In its current status, SARA is more a research tool than an intervention tool. Therefore, the interaction with the tool has not been exploited to its maximum.

The former results serve as a guideline for future research in the area, in particular the outcome from the NPR-DECT study. More research is needed to improve, or come up with real-time NPR algorithms that allow the abstraction of certain facial regions, as well as a uniform scale for abstraction. Gaze behavior will also be assessed when categorizing abstracted facial expressions, which will shed more light on the effect of stylization on ASD.

At the end of this project, it is our goal to distribute all DECTs as an open-source tool. Moreover, based on the 
results obtained with SARA new interactive applications or tests considering HCI elements will be created, taking advantage of the NPR elements and artistic abstraction techniques. This may also help to build computer-based interventions (CBI) in the future.

Acknowledgements The SARA project (officially: Impact of NonPhotorealistic Rendering for the understanding of emotional facial expressions by children and adolescents with high-functioning Autism Spectrum Disorders) is funded by DFG - German Research Foundation (AR 892/1-1, DE 620/18-1, RA 764/4-1). Thanks to Kai Goetz for the graphics.

\section{References}

1. Center for Disease Control and Prevention: Facts About ASD. Retrieved April 06, 2015, from Center for Disease Control and Prevention. http://www.cdc.gov/ncbddd/autism/facts.html (2014)

2. Baio, J.: Prevalence of autism spectrum disorder among children aged 8 years-autism and developmental disabilities monitoring network, 11 sites, United States, 2010. Surveill. Summ. 63, 1-21 (2014)

3. Elsabbagh, M., Divan, G., Koh, Y., Kim, Y., Kauchali, S., Marcín, C., Montiel-Nava, C., Patel, V., Paula, C., Wang, C., Yasamy, M., Fombonne, E.: Global prevalence of autism and other pervasive developmental disorders. Autism Res. 5(3), 160-179 (2012)

4. Saemundsen, E., Magnússon, P., Georgsdóttir, I., Egilsson, E., Rafnsson, V.: Prevalence of autism spectrum disorders in an icelandic birth cohort. BMJ Open. 3(6), e002748 (2013)

5. Chita-Tegmark, M.: Attention allocation in ASD: a review and meta-analysis of eye-tracking studies. Rev. J. Autism Dev. Disord. 3(3), 209-223 (2016)

6. Uljarevic, M., Hamilton, A.: Recognition of emotions in autism: a formal meta-analysis. J. Autism Dev. Disord. 43(7), 1517-1526 (2013)

7. Whyte, E., Smyth, J., Scherf, S.: Designing serious game interventions for individuals with autism. J. Autism Dev. Disord. 45(12), 3820-3831 (2014)

8. Milne, M., Luerssen, M., Lewis, T., Leibbrandt, R., Powers, D.: Designing and evaluating interactive agents as social skills tutors for children with autism spectrum disorder. In: Perez-Marin, D., Pascual-Nieto, I., (eds.) Conversational Agents and Natural Language Interaction: Techniques and Effective Practices, pp. 23-48 (2011)

9. Grawemeyer, B., Johnson, H., Brosnan, M., Ashwin, E., Benton, L.: Developing an embodied pedagogical agent with and for young people with autism spectrum disorder. In: ITS 2012. LNCS, vol. 7315, pp. 262-267. Springer, Heidelberg (2012)

10. Alcorn, A., Pain, H., Rajendran, G., Smith, T., Lemon, O., Porayska-Pomsta, K., Foster, M.E., Avramides, K., Frauenberger, C., Bernardini, S.: Social communication between virtual characters and children with autism. In: Artificial Intelligence in Education: 15th International Conference, pp. 7-14 (2011)

11. Serret, S., Hun, S., Iakimova, G., Lozada, J., Anastassova, M., Santos, A., Vesperini, S., Askenazy, F.: Facing the challenge of teaching emotions to individuals with low- and high-functioning autism using a new serious game: a pilot study. Mol. Autism 5(37) (2014). http://www.jestimule.com

12. Alves, S., Marques, A., Queirós, C., Orvalho, V.: LIFEisGAME prototype: a serious game about emotions for children with autism spectrum disorders. PsychNol. J. 11(3), 191-211 (2013)

13. Rice, L., Wall, C., Fogel, A., Shic, F.: Computer-assisted face processing instruction improves emotion recognition, mentalizing, and social skills in students with ASD. J. Autism Dev. Disord. 45(7), 1-11 (2015)

14. Tanaka, J.W., Wolf, J.M., Klaiman, C., Koenig, K., Cockburn, J., Herlihy, L., Brown, C., Stahl, S., Kaiser, M.D., Schultz, R.T.: Using computerized games to teach face recognition skills to children with autism spectrum disorder: the let's face it! program. J. Child Psychol. Psychiatry 51(8), 944-952 (2010)

15. American Psychiatric Association: Diagnostic and Statistical Manual of Mental Disorders: DSM-5, 5th edn. American Psychiatric Publishing, Arlington (2013)

16. World Health Organization: The ICD-10 Classification of Mental and Behavioural Disorders: Diagnostic Criteria for Research, 10th edn. Geneva: World Health Organization, Switzerland (1993)

17. Krautheim, B.: The influence of alexithymia on gaze behavior patterns in male adolescents with autism spectrum disorder. Master's thesis, Albert-Ludwigs-Universität Freiburg. Institut für Psychologie (2015)

18. Kirchner, J.C., Hatri, A., Heekeren, H.R., Dziobek, I.: Autistic symptomatology, face processing abilities, and eye fixation patterns. J. Autism Dev. Disord. 41(2), 158-167 (2011)

19. Klin, A., Jones, W., Schultz, R., Volkmar, F., Cohen, D.: Visual fixation patterns during viewing of naturalistic social situations as predictors of social competence in individuals with autism. Arch. Gen. Psychiatry 59(9), 809-816 (2002)

20. Senju, A., Johnson, M.H.: Atypical eye contact in autism: models, mechanisms and development. Neurosci. Biobehav. Rev. 33(8), 1204-1214 (2009)

21. Sawyer, A.C.P., Williamson, P., Young, R.L.: Can gaze avoidance explain why individuals with asperger's syndrome can't recognise emotions from facial expressions? J. Autism Dev. Disord. 42(4), 606-618 (2012)

22. Bird, G., Press, C., Richardson, D.: The role of alexithymia in reduced eye-fixation in autism spectrum conditions. J. Autism Dev. disord. 41(11), 1556-1564 (2011)

23. Bird, G., Cook, R.: Mixed emotions: the contribution of alexithymia to the emotional symptoms of autism. Transl. Psychiatry 3, e285 (2013)

24. Rauh, R., Schaller, U.: Categorical perception of emotional facial expressions in video clips with natural and artificial actors: a pilot study. Tech. Rep. ALU-KJPP-2009-001, University of Freiburg (2009)

25. Arellano, D., Helzle, V., Schaller, U., Rauh, R.: Animated faces, abstractions and autism. In: Intelligent Virtual Agents 2014, Lecture Notes in Computer Science, vol. 8637, pp. 22-25 (2014)

26. Helzle, V., Biehn, C., Schlömer, T., Linner, F.: Adaptable setup for performance driven facial animation. In: ACM SIGGRAPH 2004 Sketches, p. 54 (2004)

27. Ekman, P., Friesen, W., Hager, J.: The Facial Action Coding System. Weidenfeld \& Nicolson, London (2002)

28. Peirce, J.W.: Generating stimuli for neuroscience using PsychoPy. Front. Neuroinform. 2(10) (2008)

29. Arellano, D., Schaller, U.M., Rauh, R., Helzle, V., Spicker, M., Deussen, O.: Intelligent Virtual Agents: 15th International Conference, IVA 2015, Delft, The Netherlands, August 26-28, 2015, Proceedings, chap. On the Trail of Facial Processing in Autism Spectrum Disorders, pp. 432-441. Springer International Publishing, Cham (2015)

30. Law Smith, M.J., Montagne, B., Perrett, D.I., Gill, M., Gallagher, L.: Detecting subtle facial emotion recognition deficits in highfunctioning autism using dynamic stimuli of varying intensities. Neuropsychologia 48(9), 2777-2781 (2010)

31. Lozier, L.M., Vanmeter, J.W., Marsh, A.: Impairments in facial affect recognition associated with autism spectrum disorders: A meta-analysis. Dev. Psychopathol. 26(4 Pt 1), 933-945 (2014) 
32. Gooch, B., Gooch, A.: Non-Photorealistic Rendering. A.K. Peters, Natick (2001)

33. DeCarlo, D., Santella, A.: Stylization and abstraction of photographs. ACM Trans. Graph. 21(3), 769-776 (2002)

34. Gooch, B., Reinhard, E., Gooch, A.: Human facial illustrations: creation and psychophysical evaluation. ACM Trans. Graph. 23(1), 27-44 (2004)

35. Spicker, M., Arellano, D., Schaller, U., Rauh, R., Helzle, V., Deussen, O.: Emotion recognition in autism spectrum disorder: Does stylization help? In: Proceedings of the ACM Symposium on Applied Perception, SAP '16, pp. 97-104. ACM (2016)

36. Sifneos, P.: The prevalence of alexithymic characteristics in psychosomatic patients. Psychother. Psychosom. 22, 255-262 (1973)
37. Weiß, R.H.: Grundintelligenztest Skala 2 - Revision - (CFT 20-R). Hogrefe, Göttingen (2006)

38. Holmqvist, K., Nyström, M., Andersson, R., Dewhurst, R., Jarodzka, H., Van de Weijer, J. (eds.): Eye Tracking: A Comprehensive Guide to Methods and Measures. Oxford University Press, Oxford (2011)

39. Kupfer, J., Brosig, B., Brähler, E.: TAS-26: Toronto-Alexithymie-Skala-26 (deutsche Version). Manual. Hogrefe Verlag GmbH \& Co, Göttingen (2001)

40. Bermond, B., Vorst, H.C., Vingerhoets, A.J., Gerritsen, W.: The amsterdam alexithymia scale: its psychometric values and correlations with other personality traits. Psychother. Psychosom. 68(5), 241-251 (1999) 